\title{
User selection protocol in DF cooperative networks with hybrid TSR-PSR protocol based full-duplex energy harvesting over rayleigh fading channel: system performance analysis
}

\author{
Phu Tran Tin ${ }^{1}$, Le Anh Vu², Tan N. Nguyen ${ }^{3}$, Thanh-Long Nguyen ${ }^{4}$ \\ ${ }^{1}$ Faculty of Electronics Technology, Industrial University of Ho Chi Minh City, Vietnam. \\ ${ }^{2}$ Optoelectronics Research Group, Faculty of Electrical and Electronics Engineering, \\ Ton Duc Thang University, Vietnam \\ ${ }^{3}$ Wireless Communications Research Group, Faculty of Electrical \& Electronics Engineering, \\ Ton Duc Thang University, Vietnam \\ ${ }^{4}$ Center for Information Technology, Ho Chi Minh City University of Food Industry, Vietnam
}

\begin{tabular}{l} 
Article Info \\
\hline Article history: \\
Received Jul 12, 2018 \\
Revised Oct 20, 2018 \\
Accepted Nov 2, 2018 \\
\hline Keywords: \\
Decode-and-forward (DF) \\
Energy harvesting (EH) \\
Full-duplex (FD) \\
Relaying network \\
User selection
\end{tabular}

User selection

\begin{abstract}
Cooperative communication has been recently proposed in wireless communication systems for exploring the inherent spatial diversity in relay channels. In this work, we investigate the system performance of the energy harvesting full-duplex (FD) decode-and-forward (DF) hybrid time switchingpower splitting relaying TSR-PSR (TPSR) protocol relaying network. In the selection scheme, the best user selection protocol is proposed and investigated. Mainly we derive the closed-form expression for the outage probability, system throughput and the symbol error rate (SER) of the system. Numerical results are also presented by the Monte Carlo simulation to validate the theoretical analysis in connection with the all possible parameters in the comparison between TSPR, TSR and PSR cases. The research results show that TPSR case is better than the others in term of outage probability and SER.
\end{abstract}

Copyright $@ 2019$ Institute of Advanced Engineering and Science. All rights reserved.

\section{Corresponding Author:}

Le Anh $\mathrm{Vu}$,

Optoelectronics Research Group

Faculty of Electrical and Electronics Engineering

Ton Duc Thang University

Ho Chi Minh City, Vietnam.

Email: leanhvu@tdtu.edu.vn

\section{INTRODUCTION}

In recent years, the wireless transmission has experienced rapid development. Relaying has been proved to be an efficient way to extend the coverage area of wireless networks and increase transmission reliability without additional transmit power at the transmitters. However, the forwarding operations at the relay still require extra energy. For relay nodes powered by power limited batteries instead of the regular power grid, such as mobile devices, such extra energy consumption may cause serious concerns. Recently, harvesting energy from ambient radio-frequency (RF) energy was proposed, and it has been well studied. The idea that receiving information and harvesting energy simultaneously is not only appealing but also worth further investigating. The recent works suggested that data transmitting and battery charging could be fulfilled at the same time, and therefore, the combining of energy harvesting module and relay could be an available solution of extra energy consumption problem at the relay [1-6].

Recently, deploying energy harvesting (EH) relays that utilize the energy collected from the source signal for data transfer has received considerable attention. With EH capabilities, relays can be installed conveniently without wiring cost and the need for battery replacement. Besides, substantial transmission power can be saved because the inter-node distance is shortened using multihop transmissions. However, information 
relaying consumes additional resources (i.e., time and bandwidth) compared to the direct transmission and hence some early work has been conducted focusing on assessing the feasibility of EH relays. The gain offered by the EH relay based on time switching relaying (TSR) is analyzed theoretically in [7] for both decode-andforward (DF) and amplify-and-forward (AF) relaying approaches with a significant gain. The EH relay based on DF is also considered in [8], where the relay determines whether to perform EH or information relaying before the source transmission according to a greedy policy. On the other hand, an analytical framework is proposed in [9] to evaluate the performance of the EH relay based on AF for both TSR and power splitting relaying (PSR)under the effect of the critical system parameters such as noise variances, source to relay distance, transmission rate, and energy conversion efficiency. Moreover, relay selection (RS) is a practical approach to balance the tradeoff between reliability improvement and spectral efficiency loss due to information repetition [10]. In [11], two RS schemes aiming to attain the optimal tradeoff between energy transfer and outage probability/ergodic capacity for DF relays are studied.

In this work, we propose and investigate the system performance of the energy harvesting full-duplex (FD) decode-and-forward (DF) hybrid TSR-PSR protocol relaying network. For details on this analysis, the energy, and information are transferred from the source to the relay nodes, and all channels are considered as the Rayleigh fading channels. The main contributions of the paper are summarized as follows:

1. The system model of the energy harvesting full-duplex (FD) decode-and-forward (DF) hybrid TSR-PSR protocol relaying network over the Rayleigh fading channels and the comparison between hybrid TSR-PSR (TPSR), TSR and PSR cases are proposed and investigated.

2. The closed-form expressions of the outage probability and the system throughput are derived. Moreover, the best user selection protocol is proposed and investigated.

3. The symbol Error Ratio (SER) analysis of the proposed model system is presented and demonstrated.

4. The influence of the main parameters on the system performance is demonstrated entirely by the Monte Carlo simulation.

The structure of this paper is proposed as follows. Sections II presents the system model of the relaying network. Sections III derives the system performance of the model system. Section IV provides the numerical results and some discussions. Finally, Section V concludes the paper.

\section{SYSTEM MODEL}

In this paper, the system model is the energy harvesting full-duplex (FD) decode-and-forward (DF) hybrid TSR-PSR protocol relaying network as shown in Figure 1. In this model, the information is transferred from the source $(S)$ to the multi-destination $\left(D_{i}\right)$, through energy constrained intermediate relay $(R)$. The energy harvesting and information processing of the system model with hybrid TSR-PSR protocol are proposed in Figure 2. In this scheme, $\mathrm{T}$ is the block time in which the source fully transmits the information data to the multi-destination. In the first interval time $(\alpha \mathrm{T})$, the relay harvests energy from the source signal, where $\alpha$ is the time switching factor $\alpha \in(0,1)$. In the remaining interval time $(1-\alpha) \mathrm{T}$, the relay node harvests $\rho \mathrm{P}_{\mathrm{s}}$ energy from the source and use $(1-\rho) \mathrm{P}_{\mathrm{s}}$ energy to transfer information to the multi-destination nodes, which $\rho$ is the power splitting factor $\rho \in(0,1)$. All the fading channels from $\mathrm{S}$ to $\mathrm{R}$ and $\mathrm{R}$ to $\mathrm{D}$ are proposed as the Rayleigh fading channels. More details of the analytical mathematical model of the outage probability and throughput of the system model is presented and analyzed in the following sections [12-16]. If $\alpha=0$, this scheme becomes PSP. If $\rho=0$ then it becomes the TSP protocol.

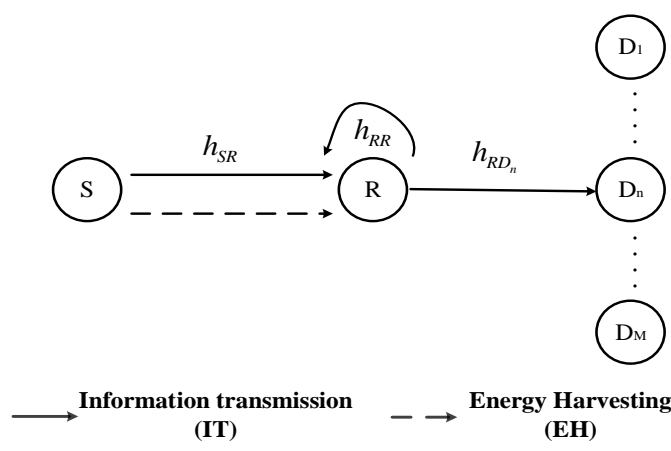

Figure 1. System model

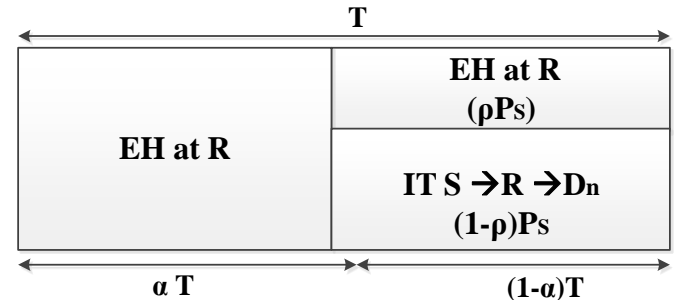

Figure 2. The power splitting protocol 


\section{THE SYSTEM PERFORMANCE}

In this section, we analyzed and demonstrated the system performance analysis of the model system [12-14].

\subsection{Energy harvesting phase}

In the first interval, the average transmitted power at the relay can be calculated as:

$$
P_{r 1}=\frac{E_{1}}{(1-\alpha) T}=\frac{\eta \alpha T P_{s}\left|h_{S R}\right|^{2}}{(1-\alpha) T}=\frac{\eta \alpha}{1-\alpha} P_{s}\left|h_{S R}\right|^{2}
$$

where $0<\eta \leq 1$ is energy conversion efficiency, $0 \leq \alpha<1$ is time switching factor, $\mathrm{P}_{\mathrm{s}}$ is the transmitted power at source, $h_{S R}$ is the source to relay channel gain, respectively.

In the second interval, once again average transmitted power at the relay can be given as:

$$
P_{r 2}=\frac{E_{2}}{(1-\alpha) T}=\frac{\eta \rho(1-\alpha) T P_{s}\left|h_{S R}\right|^{2}}{(1-\alpha) T}=\eta \rho P_{s}\left|h_{S R}\right|^{2}
$$

where $0 \leq \rho<1$ is the power splitting factor.

Finally, the amount of average transmitted power at the relay can be obtained as:

$$
P_{r}=P_{r 1}+P_{r 2}=\kappa P_{s}\left|h_{S R}\right|^{2}
$$

where we denote $\kappa=\eta \rho+\frac{\eta \alpha}{1-\alpha}$.

\subsection{Transmission phase}

The received signal at the relay can be expressed as:

$$
y_{r}=\sqrt{1-\rho} h_{S R} x_{s}+h_{R R} x_{r}+n_{r}
$$

where we denote $\mathrm{E}\left\{\left|x_{s}\right|^{2}\right\}=P_{s}, \mathrm{E}\left\{\left|x_{r}\right|^{2}\right\}=P_{r}, \mathrm{E}\{\bullet\}$ is expectation operator, $h_{R R}$ is loopback interference channel, and $\mathrm{n}_{\mathrm{r}}$ is the additive white Gaussian noise (AWGN) with variance $\mathrm{N}_{0}$.

The received signal at the $\mathrm{n}^{\text {rd }}$ destination can be formulated as:

$$
y_{d_{n}}=h_{R D_{n}} x_{r}+n_{d_{n}}
$$

where $h_{R D n}$ is the relay to the $\mathrm{n}^{\text {rd }}$ destination channel gain, $\mathrm{n}_{\mathrm{dn}}$ is the additive white Gaussian noise (AWGN) with variance $\mathrm{N}_{0}$, and $n \in(1,2, \ldots, M)$.

In this model, we consider decode and forward protocol (DF). From (4), the signal to noise ratio (SNR) at the relay can be calculated as

$$
\gamma_{1}=\frac{(1-\rho)\left|h_{S R}\right|^{2} P_{s}}{\left|h_{R R}\right|^{2} P_{r}+N_{0}}
$$

Substituting (3) into (4) and using the fact that $\mathrm{N}_{0}<<\mathrm{P}_{\mathrm{s}}$, we have

$$
\gamma_{1}=\frac{(1-\rho)\left|h_{S R}\right|^{2} P_{s}}{\kappa P_{s}\left|h_{S R}\right|^{2}\left|h_{R R}\right|^{2}+N_{0}} \approx \frac{(1-\rho)}{\kappa\left|h_{R R}\right|^{2}}
$$

From (5) and substituting (3), the SNR at the destination can be calculated as; 
$\gamma_{2}=\frac{P_{r}\left|h_{R D_{n}}\right|^{2}}{N_{0}}=\frac{\kappa P_{s}\left|h_{S R}\right|^{2}\left|h_{R D_{n}}\right|^{2}}{N_{0}}$

Finally, the end to end SNR of the proposed system can be obtain as:

$$
\gamma_{e 2 e}=\min \left(\gamma_{1}, \gamma_{2}\right)
$$

In this analysis, please note that all of the channel belong to Rayleigh fading channels.

Remark 1: The best user selection protocol. as follows:

From (9), we propose the optimal user selection protocol in which the best selection user is selected

$$
\omega_{2}=\max _{n=1,2, \ldots, M}\left(\left|h_{R D_{n}}\right|^{2}\right)
$$

In [17], the Cumulative Distribution Function (CDF) of $\omega_{2}$ can be given by the following

$$
F_{\omega_{2}}(y)=\sum_{p=0}^{M}(-1)^{p} C_{M}^{p} \times e^{-p y / \lambda_{2}}
$$

where $\lambda_{2}$ is the mean of $\mathrm{RV} \omega_{2}$, and $C_{M}^{p}=\frac{M !}{p !(M-p) !}$.

Then, the corresponding Probability Density Function (PDF) can be obtained by the following

$$
f_{\omega_{2}}(y)=\frac{1}{\lambda_{2}} \sum_{p=0}^{M-1}(-1)^{p} C_{M-1}^{p} M \times e^{-(p+1) y / \lambda_{2}}
$$

\section{Remark 2: Outage probability (OP).}

From (9), the OP of DF system can be expressed as:

$$
\begin{aligned}
O P & =\operatorname{Pr}\left(\gamma_{e 2 e}<\gamma_{t h}\right)=\operatorname{Pr}\left[\min \left(\gamma_{1}, \gamma_{2}\right)<\gamma_{t h}\right] \\
& =\operatorname{Pr}\left[\min \left(\frac{(1-\rho)}{\kappa\left|h_{R R}\right|^{2}}, \frac{\kappa P_{s}\left|h_{S R}\right|^{2} \max _{1 \leq n \leq M}\left|h_{R D_{n}}\right|^{2}}{N_{0}}\right)<\gamma_{t h}\right] \\
& =\operatorname{Pr}\left[\min \left(\frac{(1-\rho)}{\kappa \omega}, \kappa \gamma_{0} \omega_{1} \omega_{2}\right)<\gamma_{t h}\right]
\end{aligned}
$$

where we denote $\omega=\left|h_{R R}\right|^{2}, \omega_{1}=\left|h_{S R}\right|^{2}, \omega_{2}=\max _{1 \leq n \leq M}\left|h_{R D_{n}}\right|^{2}, \gamma_{0}=\frac{P_{s}}{N_{0}}, \gamma_{t h}=2^{2 R}-1$, and R is the source rate.

From (13), the OP can be rewritten as the following

$$
O P=1-\operatorname{Pr}\left[\frac{(1-\rho)}{\kappa \omega} \geq \gamma_{t h}\right] \operatorname{Pr}\left(\kappa \gamma_{0} \omega_{1} \omega_{2} \geq \gamma_{t h}\right)
$$

In (14), we denote

$$
I_{1}=\operatorname{Pr}\left[\frac{(1-\rho)}{\kappa \omega} \geq \gamma_{t h}\right]=\operatorname{Pr}\left[\omega \leq \frac{(1-\rho)}{\kappa \gamma_{t h}}\right]=1-e^{-\frac{(1-\rho)}{\kappa \gamma_{t h} \lambda}}
$$

where $\lambda$ is the mean of RV $\omega$. And 


$$
I_{2}=\operatorname{Pr}\left(\kappa \gamma_{0} \omega_{1} \omega_{2} \geq \gamma_{t h}\right)=1-\operatorname{Pr}\left(\omega_{1}<\frac{\gamma_{t h}}{\kappa \gamma_{0} \omega_{2}}\right)=1-\int_{0}^{\infty} F_{\omega_{1}}\left(\frac{\gamma_{t h}}{\kappa \gamma_{0} \omega_{2}} \mid \omega_{2}\right) f_{\omega_{2}}\left(\omega_{2}\right) d \omega_{2}
$$

Substituting (12) into (16), we have

$$
\begin{aligned}
I_{2} & =\frac{1}{\lambda_{2}} \int_{0}^{\infty} \sum_{p=0}^{M-1}(-1)^{p} C_{M-1}^{p} M \times e^{-(p+1) \omega_{2} / \lambda_{2}} \times e^{-\frac{\gamma_{t h}}{\kappa \gamma_{0} \omega_{2} \lambda_{1}}} d \omega_{2} \\
& =\sum_{p=0}^{M-1} \frac{(-1)^{p} C_{M-1}^{p} M}{\lambda_{2}} \int_{0}^{\infty} e^{-\frac{\gamma_{t h}}{\kappa \gamma_{0} \omega_{2} \lambda_{1}}} \times e^{-(p+1) \omega_{2} / \lambda_{2}} d \omega_{2}
\end{aligned}
$$

where $\lambda_{1}$ is the mean of RV $\omega_{1}$.

Applying table of integral eq $(3.324,1)$ in [18], the (17) can be reformulated as

$$
I_{2}=2 \sum_{p=0}^{M-1}(-1)^{p} C_{M-1}^{p} M \sqrt{\frac{\gamma_{t h}}{\kappa \gamma_{0} \lambda_{1} \lambda_{2}(p+1)}} \times K_{1}\left(2 \sqrt{\frac{\gamma_{t h}(p+1)}{\kappa \gamma_{0} \lambda_{1} \lambda_{2}}}\right)
$$

where $K_{v}(\bullet)$ is the modified Bessel function of the second kind and $\mathrm{v}^{\text {th }}$ order.

Substituting (16), (18) into (14), OP can be calculated as

$$
\begin{aligned}
O P & =1-\left(1-e^{-\frac{(1-\rho)}{\kappa \gamma_{t h} \lambda}}\right)\left\{2 \sum_{p=0}^{M-1}(-1)^{p} C_{M-1}^{p} M \sqrt{\frac{\gamma_{t h}}{\kappa \gamma_{0} \lambda_{1} \lambda_{2}(p+1)}} \times K_{1}\left(2 \sqrt{\frac{\gamma_{t h}(p+1)}{\kappa \gamma_{0} \lambda_{1} \lambda_{2}}}\right)\right\} \\
& =1-2 \sum_{p=0}^{M-1}(-1)^{p} C_{M-1}^{p} M \sqrt{\frac{\gamma_{t h}}{\kappa \gamma_{0} \lambda_{1} \lambda_{2}(p+1)} \times K_{1}\left(2 \sqrt{\frac{\gamma_{t h}(p+1)}{\kappa \gamma_{0} \lambda_{1} \lambda_{2}}}\right)} \\
& +2 \sum_{p=0}^{M-1}(-1)^{p} C_{M-1}^{p} M \times e^{-\frac{(1-\rho)}{\kappa \gamma_{t h} \lambda}} \times \sqrt{\frac{\gamma_{t h}}{\kappa \gamma_{0} \lambda_{1} \lambda_{2}(p+1)}} \times K_{1}\left(2 \sqrt{\frac{\gamma_{t h}(p+1)}{\kappa \gamma_{0} \lambda_{1} \lambda_{2}}}\right)
\end{aligned}
$$

\section{Remark 3: Throughput.}

$$
\tau=(1-O P) \frac{R(1-\alpha) T}{T}=(1-O P) R(1-\alpha)
$$

\section{Remark 4: The symbol Error Ratio (SER) analysis}

In this section, we obtain new expressions for the symbol Error Ratio (SER) at the destination. We first consider the outage probability, which was obtained in [19,21]. Thus, we have

$$
\begin{aligned}
& S E R=\mathrm{E}\left[\phi Q\left(\sqrt{2 \theta \gamma_{e 2 e}}\right]\right. \\
& Q(t)=\frac{1}{\sqrt{2 \pi}} \int_{t}^{\infty} e^{-x^{2} / 2} d x \text { is the Gaussian Q-function, } \omega \text { and } \theta \text { are constants which is specific for }
\end{aligned}
$$
modulation type. $(\phi, \theta)=(1,1)$ for BPSK and $(\phi, \theta)=(1,2)$ for QPSK. As a result, before obtaining the SER performance, the distribution function of $\gamma_{e 2 e}$ is expected. Then, we begin rewriting the SER expression given in (21) directly in terms of outage probability at the source by using integration, as follows

$$
S E R=\frac{\phi \sqrt{\theta}}{2 \sqrt{\pi}} \int_{0}^{\infty} \frac{e^{-\theta x}}{\sqrt{x}} F_{\gamma_{e 2 e}}(x) d x
$$


Substituting (19) into (22) and replace $\gamma_{t h}=x$, we have:

$$
\begin{aligned}
& S E R=\frac{\phi \sqrt{\theta}}{2 \sqrt{\pi}} \int_{0}^{\infty} \frac{e^{-\theta x}}{\sqrt{x}}\left\{\begin{array}{l}
1-2 \sum_{p=0}^{M-1}(-1)^{p} C_{M-1}^{p} M \sqrt{\frac{x}{\kappa \gamma_{0} \lambda_{1} \lambda_{2}(p+1)}} \times K_{1}\left(2 \sqrt{\frac{x(p+1)}{\kappa \gamma_{0} \lambda_{1} \lambda_{2}}}\right) \\
+2 \sum_{p=0}^{M-1}(-1)^{p} C_{M-1}^{p} M \times e^{-\frac{(1-\rho)}{\kappa x \lambda}} \times \sqrt{\frac{x}{\kappa \gamma_{0} \lambda_{1} \lambda_{2}(p+1)}} \times K_{1}\left(2 \sqrt{\frac{x(p+1)}{\kappa \gamma_{0} \lambda_{1} \lambda_{2}}}\right)
\end{array}\right\} d x
\end{aligned}
$$

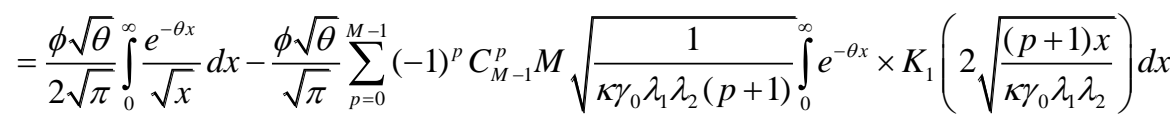

$$
\begin{aligned}
& +\frac{\phi \sqrt{\theta}}{\sqrt{\pi}} \sum_{p=0}^{M-1}(-1)^{p} C_{M-1}^{p} M \sqrt{\frac{1}{\kappa \gamma_{0} \lambda_{1} \lambda_{2}(p+1)}} \int_{0}^{\infty} e^{-\theta x} e^{-\frac{(1-\rho)}{\kappa x \lambda}} \times K_{1}\left(2 \sqrt{\frac{(p+1) x}{\kappa \gamma_{0} \lambda_{1} \lambda_{2}}}\right) d x
\end{aligned}
$$

Here we denote that

$$
J_{1}=\frac{\phi \sqrt{\theta}}{2 \sqrt{\pi}} \int_{0}^{\infty} \frac{e^{-\theta x}}{\sqrt{x}} d x
$$

Applying table of integral eq $(3.361,2)$ in [18]

We have $J_{1}=\frac{\phi}{2}$ and we denote $J_{2}$ as following

$$
J_{2}=\frac{\phi \sqrt{\theta}}{\sqrt{\pi}} \sum_{p=0}^{M-1}(-1)^{p} C_{M-1}^{p} M \sqrt{\frac{1}{\kappa \gamma_{0} \lambda_{1} \lambda_{2}(p+1)}} \int_{0}^{\infty} e^{-\theta x} \times K_{1}\left(2 \sqrt{\frac{(p+1) x}{\kappa \gamma_{0} \lambda_{1} \lambda_{2}}}\right) d x
$$

Applying table of integral eq(6.614,5) in [18], we have

$$
\begin{aligned}
& J_{2}=\frac{\phi}{4 \theta} \sum_{p=0}^{M-1}(-1)^{p} C_{M}^{p} \frac{M}{\kappa \gamma_{0} \lambda_{1} \lambda_{2}} \times e^{-\frac{p+1}{2 \kappa \gamma_{0} \lambda_{1} \theta} \theta}\left[K_{1}\left(\frac{p+1}{2 \kappa \gamma_{0} \lambda_{1} \lambda_{2} \theta}\right)-K_{0}\left(\frac{p+1}{2 \kappa \gamma_{0} \lambda_{1} \lambda_{2} \theta}\right)\right] \\
& J_{3}=\frac{\phi \sqrt{\theta}}{\sqrt{\pi}} \sum_{p=0}^{M-1}(-1)^{p} C_{M-1}^{p} M \sqrt{\frac{1}{\kappa \gamma_{0} \lambda_{1} \lambda_{2}(p+1)}} \int_{0}^{\infty} e^{-\theta x} e^{-\frac{(1-\rho)}{\kappa x \lambda}} \times K_{1}\left(2 \sqrt{\frac{(p+1) x}{\kappa \gamma_{0} \lambda_{1} \lambda_{2}}}\right) d x
\end{aligned}
$$

Finally, SER of the system model can be calculated as

$$
S E R=J_{1}-J_{2}+J_{3}
$$

\section{NUMERICAL RESULTS AND DISCUSSION}

In this section, the Monte Carlo simulation is used for validating the analytical expression in the above section. We consider a network with one source, one relay, and multi-destination, where source-relay and relaydestination distances are both normalized to unit value. Moreover, we investigate and compare TSR, PSR and TPSR cases in the connection of all possible system parameters.

The effect of $\eta$ on the outage probability and system throughput of the proposed relay network system in the comparison between TSR, PSR, TPSR cases are shown in Figure 3 and 4. In these Figures, we set the main system parameters as $P_{s} / N_{0}=10 \mathrm{~dB}, R=0.5 \mathrm{bps}$, and $\mathrm{M}=2$. From the results, the analytical and the simulation results match for all possible values $\mathrm{P}_{\mathrm{s}} / \mathrm{N}_{0}$. Figure 3 shows that the outage probability has a considerable decrease while $\eta$ increases from 0 to 1 . On another hand, system throughput significantly increases in connection with increasing of $\eta$ from 0 to 1 . The research results show that the outage probability and the system throughput have the better value in the TPSR case in comparison with the others cases. 


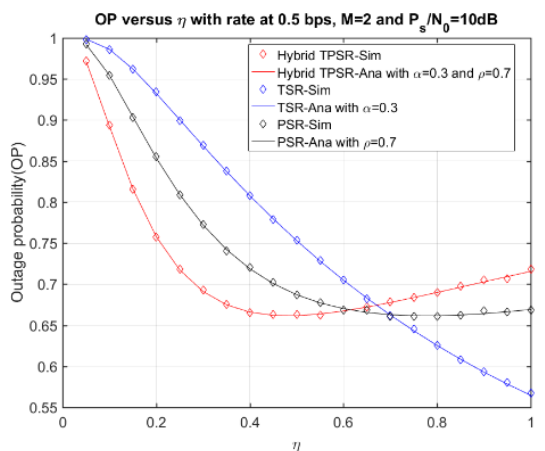

Figure 3. Outage probability versus $\eta$

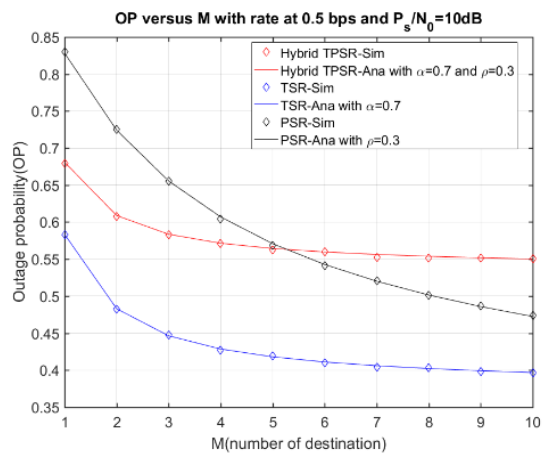

Figure 5. Outage probability versus $\mathrm{M}$

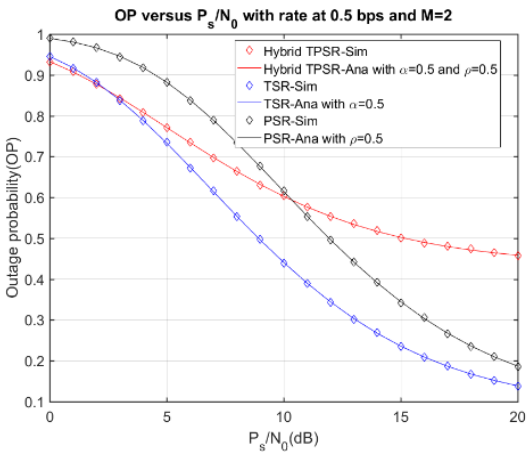

Figure 7. Outage probability versus $\mathrm{P}_{\mathrm{s}} / \mathrm{N}_{0}$

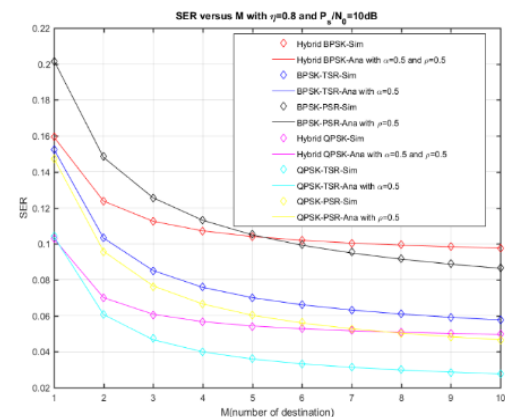

Figure 9. Comparison SER versus M

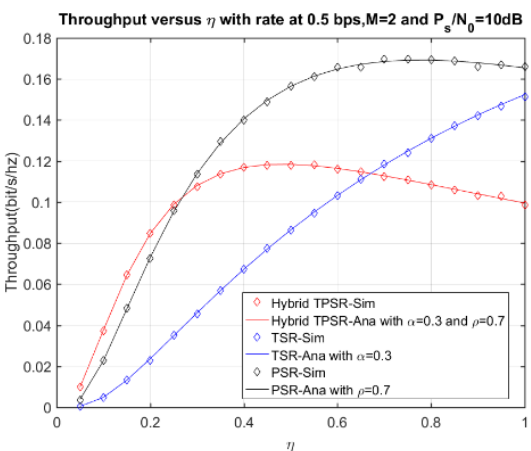

Figure 4. Throughput versus $\eta$

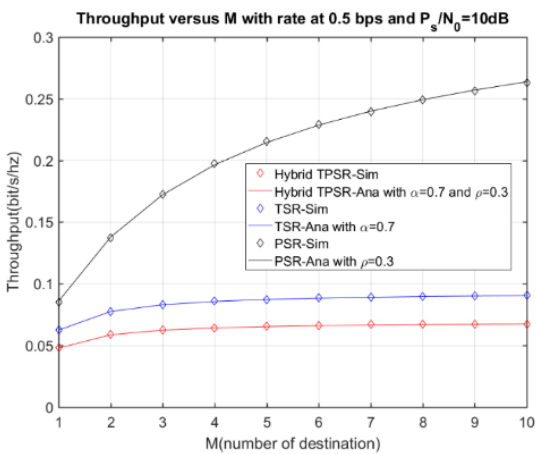

Figure 6. Throughput versus M

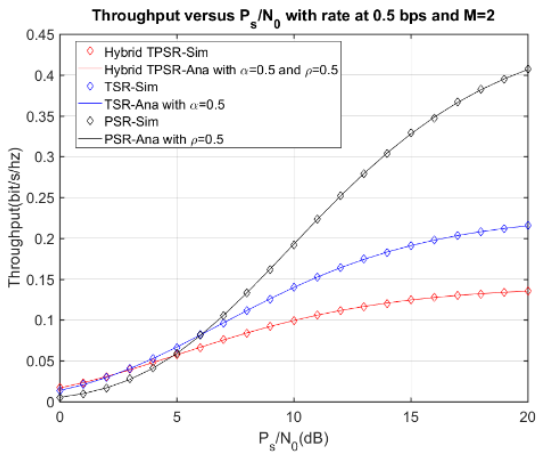

Figure 8. Throughput versus $\mathrm{P}_{\mathrm{S}} / \mathrm{N}_{0}$

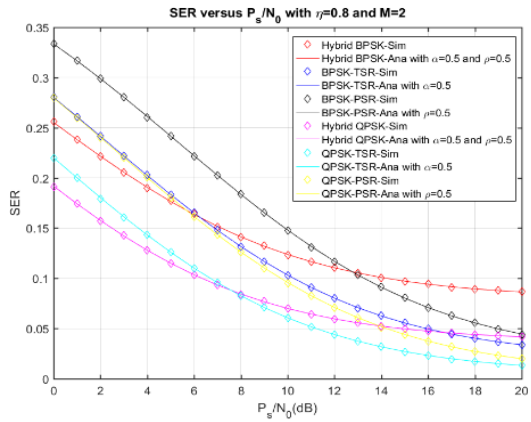

Figure 10. Comparison SER versus $\mathrm{P}_{\mathrm{s}} / \mathrm{N}_{0}$ 
Finally, Figures 9 and 10 plot the effect of $\mathrm{M}$ and $\mathrm{P}_{\mathrm{s}} / \mathrm{N}_{0}$ on SER in the comparison between TSR, PSR, TSPR cases with the main parameters of $\mathrm{P}_{\mathrm{s}} / \mathrm{N}_{0}=10, \eta=0.8$, and $\mathrm{M}=2$. The SER decreases while $\mathrm{M}$ and $\mathrm{P}_{\mathrm{s}} / \mathrm{N}_{0}$ increases from 1 to 10 and 0 to 20, respectively. The results show that all simulation and analytical results are matched well with each other. Moreover, SER in case TSPR is better than the remaining cases.

Furthermore, the outage probability and system throughput of the proposed system versus $\mathrm{M}$ are illustrated in the Figure 5 and 6 with $P_{s} / N_{0}=10 \mathrm{~dB}$ and $\mathrm{R}=0.5 \mathrm{bps}$. From the results, we show that the outage probability increases and the system throughput decrease remarkably with increasing $\mathrm{M}$ from 0 to 10 . On the same way, the outage probability and the system throughput versus $P_{s} / N_{0}$ with $R=0.5$ bps and $M=2$ as shown in Figures. 7 and 8. All the analytical and simulation results agree well with each other.

\section{CONCLUSION}

In this work, we investigate the system performance of the energy harvesting full-duplex (FD) decodeand-forward (DF) hybrid TSR-PSR protocol relaying network. In the selection scheme, the best user selection protocol is proposed and investigated. Mainly we derive the closed-form expression for the outage probability, system throughput and the symbol error rate (SER) of the system. Numerical results are also presented by the Monter Carlo simulation to validate the theoretical analysis in connection with the all possible parameters in the comparison between TPSR, TSR and PSR cases. The research results show that TPSR case is better than the others in term of outage probability and SER. This paper provides the novel recommendation for the communication relaying network in the near future.

\section{REFERENCES}

[1] Chen, He, Chao Zhai, Yonghui Li, and Branka Vucetic. "Cooperative Strategies for Wireless-Powered Communications: An Overview." IEEE Wireless Communications 25, no. 4 (08 2018): 112-19. doi:10.1109/mwc.2017.1700245.

[2] Bi, S., Ho, C. K., \& Zhang, R. Wireless powered communication: Opportunities and challenges. IEEE Communications Magazine, 2015; 53: 117-125.

[3] Niyato, D., Kim, D. I., Maso, M., \& Han, Z. Wireless Powered Communication Networks: Research Directions and Technological Approaches. IEEE Wireless Communications, 2017, 2-11.

[4] Yu, H., Lee, H., \& Jeon, H. (2017, 10). What is 5G? Emerging 5G Mobile Services and Network Requirements. Sustainability, 2017, 9, 1848.

[5] C. R. Valenta and G. D. Durgin, "Harvesting wireless power: Survey of energy-harvester conversion efficiency in far-field, wireless power transfer systems," IEEE Microw. Mag., 2014; 15(4): 108-120.

[6] Lai, Xiazhi, Wanxin Zou, Xutao Li, and Lisheng Fan. "Multiuser Energy Harvesting Relaying System with Direct Links." IET Communications 11, no. 12 (08, 2017): 1846-852. doi:10.1049/iet-com.2016.1288.

[7] Kawabata, Hiroki, and Koji Ishibashi. "RF Energy Powered Feedback-aided Cooperation." 2014 IEEE 25th Annual International Symposium on Personal, Indoor, and Mobile Radio Communication (PIMRC), 092014. doi:10.1109/pimrc.2014.7136211.

[8] Krikidis, Ioannis, Stelios Timotheou, and Shigenobu Sasaki. "RF Energy Transfer for Cooperative Networks: Data Relaying or Energy Harvesting?" IEEE Communications Letters 16, no. 11 (11 2012): 1772-775. doi:10.1109/lcomm.2012.091712.121395.

[9] Nasir, Ali A., Xiangyun Zhou, Salman Durrani, and Rodney A. Kennedy. "Relaying Protocols for Wireless Energy Harvesting and Information Processing." IEEE Transactions on Wireless Communications 12, no. 7 (07 2013): 3622 636. doi:10.1109/twc.2013.062413.122042.

[10] Laneman, J.n., D.n.c. Tse, and G.w. Wornell. "Cooperative Diversity in Wireless Networks: Efficient Protocols and Outage Behavior." IEEE Transactions on Information Theory 50, no. 12 (12 2004): 3062-080. doi:10.1109/tit.2004.838089.

[11] Michalopoulos, Diomidis S., Himal A. Suraweera, and Robert Schober. "Relay Selection for Simultaneous Information Transmission and Wireless Energy Transfer: A Tradeoff Perspective." IEEE Journal on Selected Areas in Communications, 2015, 1. doi:10.1109/jsac.2015.2391771.

[12] Tan N. Nguyen, T.H.Q.Minh, Phuong T. Tran and Miroslav Voznak. Energy Harvesting over Rician Fading Channel: A Performance Analysis for Half-Duplex Bidirectional Sensor Networks under Hardware Impairments. Sensors, 2018, 18

[13] Tan N. Nguyen, T.H.Q.Minh, Phuong T. Tran and Miroslav Voznak. Adaptive Energy Harvesting Relaying Protocol for Two-Way Half Duplex System Network over Rician Fading Channel. Wireless Communications and Mobile Computing, 2018.

[14] Tin, Phu Tran, Tran Hoang Quang Minh, Tan N. Nguyen, and Miroslav Voznak. "System Performance Analysis of Half-Duplex Relay Network over Rician Fading Channel." TELKOMNIKA Telecommunication, Computing, Electronics and Control, 16, no. 1 (02, 2018): 189. doi:10.12928/telkomnika.v16i1.7491.

[15] Rashid, Tarique, Sunil Kumar, Akshay Verma, Prateek Raj Gautam, and Arvind Kumar. "Pm-EEMRP: Postural Movement Based Energy Efficient Multi-hop Routing Protocol for Intra Wireless Body Sensor Network (IntraWBSN)." TELKOMNIKA Telecommunication, Computing, Electronics and Control 16, no. 1 (02, 2018): 166. doi:10.12928/telkomnika.v16i1.7318.

User selection protocol in DF cooperative networks with hybrid TSR-PSR protocol based... (Phu Tran Tin) 
[16] A. F. Morabito, "Power Synthesis of Mask-Constrained Shaped Beams Through Maximally-Sparse Planar Arrays," Telkomnika (Telecommunication Computing Electronics and Control), 2016; 14(4): 1217-1219.

[17] Nguyen, T. N., T. T. Duy, G.-T. Luu, P. T. Tran, and M. Voznak. "Energy Harvesting-based Spectrum Access with Incremental Cooperation, Relay Selection and Hardware Noises." Radioengineering 26, no. 1 (04, 2017): 240-50. doi:10.13164/re.2017.0240.

[18] Table of Integrals, Series, and Products, 2015

[19] Mckay, Matthew R., Alex J. Grant, and Iain B. Collings. "Performance Analysis of MIMO-MRC in DoubleCorrelated Rayleigh Environments." IEEE Transactions on Communications 55, no. 3 (03 2007): 497-507. doi:10.1109/tcomm.2007.892450.

[20] Tran Hoang Quang Minh. "Hybrid Time-Power Switching Protocol of Energy Harvesting Bidirectional Relaying Network: Throughput and Ergodic Capacity Analysis." TELKOMNIKA Telecommunication, Computing, Electronics and Control, 16, no. 5 (10, 2018): 189. http://dx.doi.org/10.12928/telkomnika.v16i5.9118. 